\title{
Strontium Thiogallate: An Efficient Green Emitting Phosphor for UV LEDs
}

\author{
N. D. Meshram1 ${ }^{*}$, P. J. Yadav² \\ ${ }^{1}$ Kamla Nehru Mahavidyalaya, Sakkardara, Nagpur, India \\ ${ }^{2}$ Shri Mathuradas Mohota College of Science, Sakkardara, India \\ Email: *meshramnileshsd@gmail.com
}

How to cite this paper: Meshram, N.D. and Yadav, P.J. (2018) Strontium Thiogallate: An Efficient Green Emitting Phosphor for UV LEDs. Journal of Applied Mathematics and Physics, 6, 663-666. https://doi.org/10.4236/jamp.2018.64059

Received: January 5, 2018

Accepted: April 9, 2018

Published: April 12, 2018

\begin{abstract}
In this paper we have reported green emission for europium activated strontium thiogallate. The phosphor is prepared by the precipitation method followed by heating at reducing atmosphere produced by the burning charcoal and characterized using XRD, PL and EL techniques. The phosphor $\mathrm{SrGa}_{2} \mathrm{~S}_{4}: \mathrm{Eu}^{2+}$ gives intense green emission at $525 \mathrm{~nm}$ when excited by $400 \mathrm{~nm}$ wavelength. LEDs were also fabricated by coating europium activated strontium thiogallate on $390 \mathrm{~nm}$ LED chip. Intense green LEDs were obtained which can be useful for new LED applications. This phosphor can also be a prominent green component for the fabrication of white LEDs from UV diodes.
\end{abstract}

\section{Keywords}

Luminescence, Thiogallate, SSL, UV LED etc.

\section{Introduction}

Commercially available InGaN-based blue and green light-emitting diodes (LEDs) and laser diodes (LDs) have been successfully fabricated on sapphire or SiC substrates. [1] [2] [3] Solid state lighting based on high-brightness LEDs has emerged as a new potentially revolutionary technology that could save up to half of energy used for lighting applications. Compared with the traditional lighting, white LED has the following advantages; small (use of many kinds of associations and flexible array device), not easy to damage (bear shaking), long lifetime (more than 100,000 hours), low power consumption and low pollution. Thus, White LED would be the most important light source in the 21st century [4].

White LEDs, based on blue LED chips coated with a yellow emitting phosphor (YAG:Ce), were first reported in 1997 [5]. The blue chip/YAG:Ce system has 
many advantages. But the lamps fabricated in this manner give a poor colour rendering because the resulting light is typically deficient in the green and red colours. There are two approaches which are being followed to overcome this problem. In the first approach, white LEDs are made by coating near ultraviolet (n-UV) emitting LED with a mixture of high efficiency red, green and blue emitting phosphors [6], analogous to the fluorescent lamp. This method yields lamps with better colour rendition. Addition of a yellow emitting phosphor improves colour rendition index (CRI) further. Recently, much progress has been made for the emission efficiency of LED chips in the near UV-to-deep blue range [7] [8] [9] [10]. However, cost and light output wise, near UV chips are much inferior to blue chips. In this paper, we propose an efficient green europium activated strontium thiogallate phosphor as the green candidate to obtain white LEDs from UV diodes.

\section{Experimental}

A phosphor comprising strontium thiogallate doped with europium is produced by precipitation followed by reduction in reducing atmosphere produced by burning charcoal. $2.4 \mathrm{~g}$ of sulphur is dissolved in $15 \mathrm{ml}$ of hydrazine hydrate, $\mathrm{N}_{2} \mathrm{H}_{4} \mathrm{H}_{2} \mathrm{O}$. A solution of $4 \mathrm{~g}$ of strontium chloride, $\mathrm{SrCl}_{2} 6 \mathrm{H}_{2} \mathrm{O}$, in $25 \mathrm{ml}$ of water is then added followed by the addition of a solution of 5.28 gm of gallium chloride in $20 \mathrm{ml}$ of water. The mixture is stirred for a further 30 minutes and the resultant precipitate is removed by filtration, washed with water and dried at $110^{\circ} \mathrm{C}$. $0.15 \mathrm{gm}$ of europium acetate, $\mathrm{Eu}\left(\mathrm{CH}_{3} \mathrm{CO}_{2}\right)_{3} 4 \mathrm{H}_{2} \mathrm{O}$, and 0.026 gm of sodium chloride are slurried with the dried precipitate using 2-propanol. This is dried and the mixture sintered at $900^{\circ} \mathrm{C}$ under reducing atmosphere for one hour. The resultant phosphor gives bright green emission when excited at 390 $\mathrm{nm}$ excitation wavelength.X-ray diffraction patterns were recorded on Philips PANalytical X'pert Pro diffractometer. Photoluminescence (PL) spectra in the spectral range 220 - $700 \mathrm{~nm}$ were recorded at room temperature on Hitachi F-7000 spectro-fluorimeter with spectral slit widths of $1 \mathrm{~nm}$.

The phosphor in desired quantity was dispersed in a transparent silicone resin (Wells Electronic Materials Company, 5012-2A and 5012-2B), and LED was then fabricated by coating the near UV LED chip ( CREE $390 \mathrm{~nm}, 300$ micron) with the epoxy resin. The electroluminescence (EL) spectra, colour temperature, CIE chromaticity coordinates, CRI and lumen output at room temperature were measured using a $300 \mathrm{~mm}$ integrating sphere and lumen meter (Hangzhou Zhongwei Photoelectricity Company ZVision ZWL 3900). The measurements were carried out at $3.2 \mathrm{~V}, 20 \mathrm{~mA}$.

Confirm that you have the correct template for your paper size. This template has been tailored for output on the custom paper size $(21 \mathrm{~cm} * 28.5 \mathrm{~cm})$.

\section{Results and Discussion}

Europium activated strontium thiogallate phosphor exhibit intense green emis- 
sion upon excitation of $390 \mathrm{~nm}$ wavelength. Green emitting LEDs can be prepared by coating europium activated strontium thiogallate phosphor on $390 \mathrm{~nm}$ diode.

The PL characteristic of europium activated strontium thiogallate phosphor is shown in Figure 1, the excitation and emission spectra gives the strong resemblance with the literature results. The phosphor exhibits broad excitation spectra between $300-500 \mathrm{~nm}$ wavelength range. The emission spectra give an intense band at $530 \mathrm{~nm}$ wavelength when excited by $390 \mathrm{~nm}$ wavelength. The europium activated strontium thiogallate phosphor is then coated on $390 \mathrm{~nm}$ LED chips and the EL spectra were recorded.

The LED spectra as shown in Figure 2 represents the UV converted green emitting LEDs. The curve 1 represents the curve for UV $390 \mathrm{~nm}$ diode. Curve 2 3 represents the UV converted to green emitting diodes.

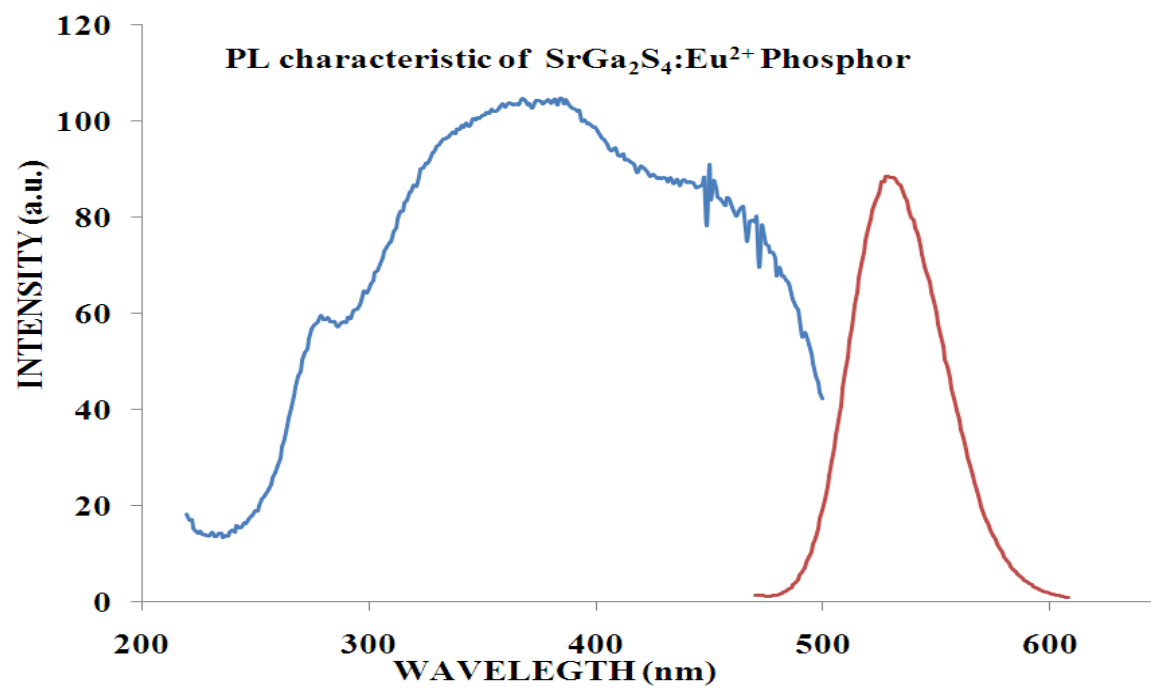

Figure 1. PL characteristic of europium activated strontium thiogallate phosphor.

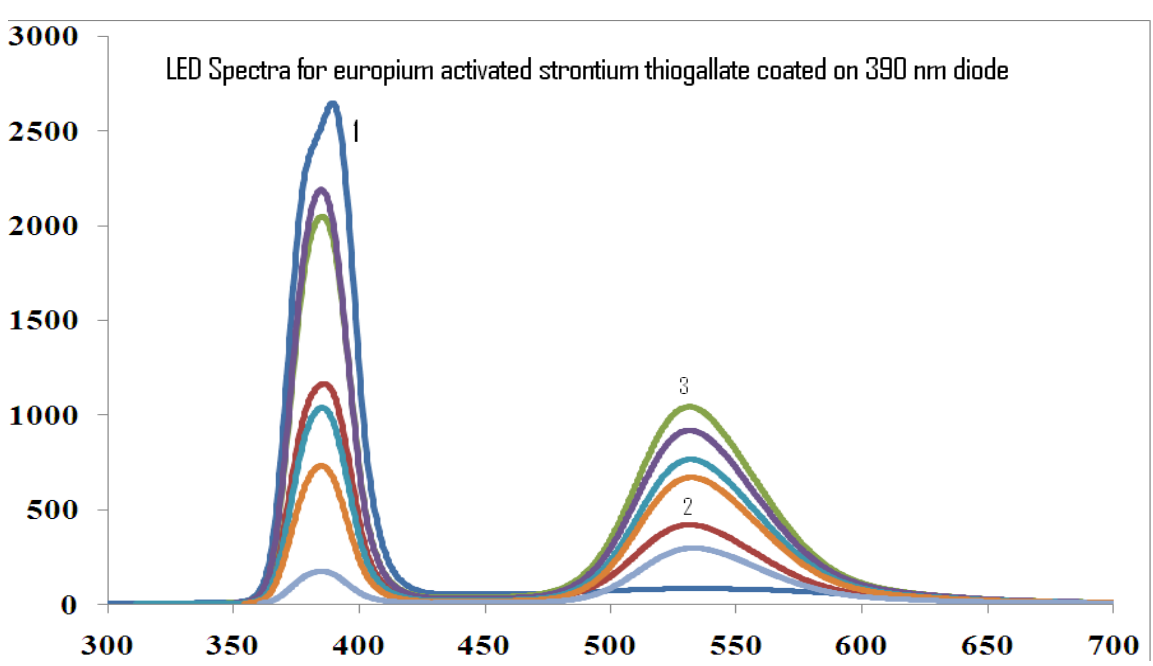

Figure 2. LED spectra for europium activated strontium thiogallate phosphor on $390 \mathrm{~nm}$ UV LED chips. 


\section{Conclusion}

The green emitting europium activated strontium thiogallate phosphor is prepared via precipitation method which helps in improving emission intensity as well as the particle size of the material. This phosphor is a strong green candidate for the fabrication of white LEDs from UV as well as blue diodes. This phosphor is also useful for fabrication of green diodes from UV LED chips.

\section{Acknowledgements}

P. J. Yadav is grateful to the Department of Science and Technology, New Delhi for the award of Women Scientist Fellowship.

\section{References}

[1] Mukai, T., Yamada, M. and Nakamura, S. (1999) Jpn. J. Appl. Phys., 38, 3976.

[2] Mack, M.P., Abare, A.C., Hansen, M., Kozodoy, P., Keller, S., Mishra, U., Coldren, L.A. and Den Baars, S.P. (1998) J. Cryst. Growth, 837, 189-190.

[3] Nakamura, S., Senoh, M., Nagahama, S.I., Iwasa, N., Yamada, T., Matsushita, T., Sugimoto, Y. and Kiyoku, H. (1997) Appl. Phys. Lett., 70, 1417.

[4] Zhang, X.M., Park, B., Choi, N., Kim, J. Kim, G.C. and Yoo, J.H. (2009) Mater.lett., 63, 700 .

[5] Nakamura, S. and Fasol, G. (1997) The Blue Laser Diode: GaN Based Light Emitters and Lasers. Spring, Heidelberg.

[6] Xu, Y., Chen, L., Li, Y., Song, G., Wang, Y., Zhuang, W. and Long, Z. (2008) Appl Phys Lett., 92, 021129.

[7] Khizar, M., Fan, Z.Y., Kim, K.H., Lin, J.Y. and Jiang, H.X. (2005) Appl Phys Lett, 86, Article ID: 173504.

[8] Chen, L., Lin, C.-C., Yeh, C.-W. and Liu, R.-S. (2010) Materials, 3, 2172.

[9] Xie, R.-J., Hirosaki, N., Li, Y.Q. and Takeda, T. (2010) Materials, 3, 3777.

[10] Ye, S., Xiao, F., Pan, Y.X., Ma, Y.Y. and Zhang, Q.Y. (2010) Mater. Sci. Engg. R, 71, 1. 